\title{
Relation Between Effective Couplings for Asymptotically Free Models
}

\author{
Reinhard Oehme ${ }^{1}$ and Wolfhart Zimmermann ${ }^{2}$ \\ 1 The Enrico Fermi Institute and the Department of Physics, The University of Chicago, \\ Chicago, IL, USA \\ 2 Max-Planck-Institut für Physik und Astrophysik, Werner-Heisenberg-Institut für Physik, \\ D-8000 München, Federal Republic of Germany
}

\begin{abstract}
For asymptotically free models with two independent couplings asymptotic expansions are constructed which express one effective coupling in terms of the other. The expansions involve powers (including fractional or irrational exponents) and logarithms. All orders of the $\beta$-functions are taken into account. The expansions found are complete in the sense that they represent solutions (exact to any order) which generalize all the solutions obtained with the $\beta$-functions approximated to second order. It is shown that higher orders are relevant since it is not possible in general to reparametrize the system such that the $\beta$-functions become polynomials of the coupling parameters. The simplifications in case of supersymmetric models are discussed.
\end{abstract}

\section{Introduction}

In this paper asymptotic properties of effective couplings will be studied for massless field theoretical models which are asymptotically free and involve two coupling constants. As example may serve a non-Abelian gauge field of coupling constant $g$ to which a Higgs field with interaction constant $\lambda$ is coupled. The effective coupling parameters $\bar{g}$ and $\bar{\lambda}$ are defined as functions of the coupling constants, a Euclidean momentum variable $k^{2}<0$ and a normalization mass $\kappa^{2}<0$. In terms of dimensionless variables,

$$
\bar{g}=\bar{g}(u, g, \lambda), \quad \bar{\lambda}=\bar{\lambda}(u, g, \lambda), \quad u=\frac{k^{2}}{\kappa^{2}} .
$$

A model is called asymptotically free if both effective couplings vanish in the limit of large Euclidean momenta [1-3]

$$
\lim _{u \rightarrow \infty} \bar{g}=0, \quad \lim _{u \rightarrow \infty} \bar{\lambda}=0 .
$$

Only solutions with bounded ratio $\bar{\lambda} / \bar{g}^{2}$ will be considered. 
The effective couplings satisfy the differential equations [4]

$$
\begin{aligned}
& u \frac{\partial \bar{g}^{2}}{\partial u}=\beta_{1}\left(\bar{g}^{2}, \bar{\lambda}\right), \\
& u \frac{\partial \bar{\lambda}}{\partial u}=\beta_{2}\left(\bar{g}^{2}, \bar{\lambda}\right) .
\end{aligned}
$$

For the asymptotic expansions of the $\beta$-functions we consider the following forms of the $\beta$-functions which should cover most applications

$$
\begin{gathered}
\beta_{1}=b_{1} g^{4}+\sum_{n=3}^{\infty} \sum_{m=0}^{n-1} b_{n-m, m} g^{2(n-m)} \lambda^{m}, \\
\beta_{2}=c_{1} \lambda^{2}+c_{2} \lambda g^{2}+c_{3} g^{4}+\sum_{n=3}^{\infty} \sum_{m=0}^{n} c_{n-m, m} \lambda^{n-m} g^{2 m} .
\end{gathered}
$$

Though there are important models with vanishing lowest order of $\beta_{1}$ we assume $b_{1}, c_{1} \neq 0$ throughout the present paper. Since terms of the form $\lambda^{m}$ are not included in $\beta_{1}$ we have

$$
\beta_{1}=0 \text { at } g=0 .
$$

Therefore, Eq. (1.3) admits the trivial solution

$$
\bar{g} \equiv 0,
$$

leaving the differential equation (1.4) for $\bar{\lambda}$ alone. This case in which the primary coupling $g$ is turned off will not be considered any further.

Of particular interest are supersymmetric gauge theories with $\lambda=h^{2}$, where $h$ describes a matter or Higgs interaction. For such models all coefficients $c_{3}$ and $c_{0 n}$ of terms $g^{2 n}$ vanish in $\beta_{2}$ so that

$$
\beta_{2}=0 \text { at } \lambda=0 \text {. }
$$

Then (1.4) allows for the trivial solution $\bar{\lambda} \equiv 0$, in which case the secondary coupling $h$ is turned off.

The ordinary differential equation

$$
\beta_{1} \frac{d \bar{\lambda}}{d \bar{g}^{2}}=\beta_{2}
$$

follows from (1.3) and (1.4) by eliminating $u$. Apart from the trivial solution (1.8) $u$ can always be eliminated since $d \bar{g}^{2} / d u \neq 0$ as a consequence of (1.2), (1.3), and (1.5) for large enough $u$. Thus except for (1.8) all asymptotically free solutions satisfy (1.11) in a sufficiently small neighborhood of $\bar{\lambda}=\bar{g}=0$. The purpose of this paper is to derive asymptotic expansions which express $\bar{\lambda}$ as a function of small values $\bar{g}$.

In case of the lowest order approximation

$$
\begin{array}{ll}
\beta_{1}=b_{1} \bar{g}^{4}, & b_{1} \neq 0, \\
\beta_{2}=c_{1} \bar{\lambda}^{2}+c_{2} \bar{\lambda} \bar{g}^{2}+c_{3} \bar{g}^{4}, & c_{1} \neq 0,
\end{array}
$$

the exact solutions of (1.11) are well-known. 
For

$$
\Delta=\left(c_{2}-b_{1}\right)^{2}-4 c_{1} c_{3}>0
$$

the solutions are

$$
\bar{\lambda}=\bar{g}^{2} \frac{\varrho_{-}+A \varrho_{+} \bar{g}^{2 \xi}}{1+A \bar{g}^{2 \xi}}=\bar{g}^{2} \frac{\varrho_{+}+B \varrho_{-} \bar{g}^{-2 \xi}}{1+B \bar{g}^{-2 \xi}}
$$

with an arbitrary constant of integration $A$ or $B$. The exponent $\xi$ is defined by

$$
\xi=-\frac{c_{1}}{b_{1}}\left(\varrho_{+}-\varrho_{-}\right) .
$$

$\varrho_{ \pm}$denotes the roots of

$$
c_{1} x^{2}+\left(c_{2}-b_{1}\right) x+c_{3}=0,
$$

with $\varrho_{+}$being the larger value

$$
\varrho_{+} \geqq \varrho_{-} .
$$

$\xi$ is non-vanishing and in sign opposite to $c_{1} / b_{1}$ if $\Delta>0$. For vanishing $A$ or $B$ there are the special solutions

$$
\bar{\lambda}_{ \pm}=\varrho_{ \pm} \bar{g}^{2}
$$

In the limit $\bar{g} \rightarrow 0$ the general solution (1.14) approaches

$$
\bar{\lambda} \rightarrow \bar{\lambda}_{-} \quad \text { if } \quad \xi>0, \quad B \neq 0
$$

and

$$
\bar{\lambda} \rightarrow \bar{\lambda}_{+} \quad \text { if } \quad \xi<0, \quad \mathrm{~A} \neq 0 .
$$

Hence for $\xi>0$ the special solution $\bar{\lambda}_{-}$which corresponds to the smaller root of (1.16) is stable while the solution $\bar{\lambda}_{+}$is unstable provided $\varrho_{+} \neq \varrho_{-}$.

For $\Delta=0$ the general solution of (1.11) and (1.12) is

$$
\bar{\lambda}=\varrho_{ \pm} \bar{g}^{2}-\frac{b_{1}}{c_{1}} \frac{\bar{g}^{2}}{\ln \bar{g}^{2}+A},
$$

where

$$
\varrho_{+}=\varrho_{-}=\frac{b_{1}-c_{2}}{2 c_{1}}
$$

is the root of (1.16). In addition there is the special solution

$$
\bar{\lambda}_{ \pm}=\varrho_{ \pm} \bar{g}^{2}
$$

which corresponds to infinite $A$.

The case $\Delta<0$ will not be considered here. It has first been observed by Gross and Wilczek that a model with $\Delta<0$ cannot be asymptotically free even if the necessary condition $b_{1}<0$ is satisfied [5]. 
The solutions based on the lowest order approximation (1.12) of the $\beta$-functions may be misleading. In particular, if the second order approximation of $\bar{\lambda}$ vanishes, the leading asymptotic behavior in $\bar{g}^{2}$ could be quite different. On the other hand it will be shown that for two independent couplings it will in general not be possible to reparametrize the system by a regular transformation such that the $\beta$-functions become polynomial. Therefore the full asymptotic expansions (1.5) and (1.6) of the $\beta$-functions will be used in this paper in order to construct expansions of $\bar{\lambda}$ in terms of powers (including fractional or irrational exponents) and logarithms of $\bar{g}^{2}$ which are valid asymptotically for small $\bar{g}$. The expansions found will be complete in the sense that they represent all possible solutions if applied to the approximated system (1.12).

We briefly state some of the results. A general solution will be constructed involving an arbitrary constant of integration and, in addition, special solutions $\bar{\lambda}_{ \pm}$which correspond to the solutions (1.18) of the approximate system. The leading term of any expansion is always $\varrho_{ \pm} \bar{g}^{2}$ provided the roots $\varrho_{ \pm}$of $(1.16)$ do not vanish.

The expansions found are only meaningful if the coefficients of the $\beta$-functions satisfy

$$
\Delta \geqq 0 \text {. }
$$

Otherwise $\bar{\lambda}$ is not real. Under the further condition

$$
b_{1}<0
$$

the expansions represent effective couplings which are asymptotically free. However, not all models satisfying $\Delta \geqq 0$ and $b_{1}<0$ are covered by the asymptotic expansions obtained. An important restriction is the positivity condition first stated by Browne, O'Raifeartaigh, and Sherry for supersymmetric models [6] ${ }^{1}$. A similar restriction in the general case excludes positive values of $\lambda$ for asymptotically free models if the roots $\varrho_{ \pm}$are negative. If $\varrho_{-}<0$ but $\varrho_{+} \geqq 0$, only an unstable mode of the system can be asymptotically free. There may be other requirements of a related nature. For instance the ratio $\lambda / g^{2}$ may for dynamical reasons be bounded, say by

$$
0<\frac{\lambda}{g^{2}} \leqq \eta
$$

If the upper bound $\eta$ is below the two roots $\varrho_{ \pm}$the model cannot be asymptotically free.

Since asymptotic freedom requires $b_{1}<0$ and $c_{1}$ is usually positive, the value of $\xi$ as defined by (1.15) is non-negative. We therefore set

in the remainder of the introduction.

$$
\xi \geqq 0
$$

Special solutions of (1.11) can be constructed in the form of power series

$$
\begin{aligned}
& \bar{\lambda}_{+}=\varrho_{+} \bar{g}^{2}+\sum_{n=2}^{\infty} a_{+n} \bar{g}^{2 n}, \\
& \bar{\lambda}_{-}=\varrho_{-} \bar{g}^{2}+\sum_{n=2}^{\infty} a_{-n} \bar{g}^{2 n},
\end{aligned}
$$

which correspond to the solutions (1.18) of the approximate system.

1 This work was generalized to models of more than two couplings in [7] 
The solution $\bar{\lambda}_{+}$always exists with uniquely determined coefficients. A unique solution $\bar{\lambda}_{-}$also exists provided $\xi$ is not a positive integer. For positive integral $\xi$ either $\bar{\lambda}_{-}$cannot be constructed or represents the general solution with arbitrary coefficient $a_{\xi+1}$.

If $\xi>0$ and not an integer the general solution $\bar{\lambda}$ involves fractional or irrational powers of $\bar{g}^{2}$. The lowest order contribution of this kind is

$$
d \bar{g}^{2(\xi+1)}
$$

with arbitrary $d$ corresponding to the constant of integration. The other coefficients are uniquely determined. The special solution $\bar{\lambda}_{-}$is obtained by setting $d=0$.

If $\xi$ is an integer, logarithms usually appear in the expansion of the general solution. For positive, integral $\xi$ the first logarithm may appear in the order $\bar{g}^{2(\xi+1)}$. In this order the general solution contains the terms

$$
a_{\xi+1} \bar{g}^{2(\xi+1)}+d_{\xi+1} \bar{g}^{2(\xi+1)} \ln \bar{g}^{2} .
$$

$a_{\xi+1}$ is arbitrary, $d_{\xi+1}$ and the other coefficients are unique. $d_{\xi+1}$ may vanish in which case the expansion becomes a power series.

For $\xi=0$ the general solution may be expanded with respect to powers of $\bar{g}^{2}$ and inverse powers of $\ln \bar{g}^{2}$. The leading terms are

$$
\bar{\lambda}=\frac{b_{1}-c_{2}}{c_{1}} \bar{g}^{2}-\frac{b_{1}}{c_{1}} \frac{\bar{g}^{2}}{\ln \bar{g}^{2}+A}+o\left(\bar{g}^{4}\right) .
$$

$A$ is an arbitrary integration constant. The coefficients of the higher order terms are unique.

The asymptotic behavior of the solutions obtained is

$$
\begin{aligned}
& \bar{\lambda} \simeq \bar{\lambda}_{-} \simeq \varrho_{-} \bar{g}^{2}, \quad \varrho_{ \pm} \neq 0, \quad \xi \geqq 0, \\
& \bar{\lambda}_{+} \simeq \varrho_{+} \bar{g}^{2},
\end{aligned}
$$

for non-vanishing roots $\varrho_{ \pm}$. Accordingly $\bar{\lambda}_{-}$is a stable solution while $\bar{\lambda}_{+}$is unstable if the roots $\varrho_{ \pm}$are different $(\xi>0)$.

For supersymmetric models these results simplify considerably. Because of $c_{3}=0$ the condition $\Delta \geqq 0$ is always satisfied. The coupling parameter $\lambda=h^{2}$ cannot be negative. The differential Eq. (1.11) always admits the trivial solution $\bar{\lambda} \equiv 0$ which corresponds to $\lambda=0$. For the interacting case the positivity condition $\lambda>0$ and the condition $b_{1}<0$ leads to the requirement

$$
c_{2}<b_{1}<0
$$

of Browne, O'Raifearthaigh, and Sherry for asymptotic freedom. It is

$$
\varrho_{-}=0, \quad \varrho_{+}=\frac{b_{1}-c_{2}}{c_{1}}>0, \quad \xi=\frac{c_{2}}{b_{1}}-1>0 .
$$

The leading behavior of the general solution is always determined by the value of $\xi$,

$$
\bar{\lambda} \simeq d \bar{g}^{2(\xi+1)}
$$


with arbitrary coefficient $d$. If $\xi$ is a positive integer, no logarithms occur and the general solution can be expanded as power series starting with the term (1.34). Apart from the trivial solution

$$
\bar{\lambda}_{-} \equiv 0
$$

there is also an unstable solution

$$
\bar{\lambda}_{+}=\varrho_{+} \bar{g}^{2}+\sum_{n=2}^{\infty} \varrho_{n} \bar{g}^{2 n}
$$

with uniquely determined coefficients.

In Sect. 2 of this paper reparametrizations in two variables will be discussed with the result that in general the $\beta$-functions cannot be made polynomial by a regular transformation. Asymptotic expansions of $\bar{\lambda}$ in terms of $\bar{g}^{2}$ are derived in Sect. 3. The special case of supersymmetric models is discussed in Sect. 4.

\section{Reparametrization in Two Variables}

We consider transformations $g^{2}, \lambda \rightarrow g^{\prime 12}, \lambda^{\prime}$ defining new coupling parameters $g^{\prime 2}, \lambda^{\prime}$ by power series expansions

$$
\begin{aligned}
g^{\prime 2} & =g^{2}+\sum_{n=2}^{\infty} \sum_{m=0}^{n-1} a_{n-m, m} g^{2(n-m)} \lambda^{m}, \\
\lambda^{\prime} & =\lambda+\sum_{n=2}^{\infty} \sum_{m=0}^{n-1} d_{n-m, m} \lambda^{n-m} g^{2 m} .
\end{aligned}
$$

In case of a single coupling parameter it has been shown by 't Hooft that the $\beta$-function can always be made polynomial by a regular reparametrization [8]. Here the corresponding problem with two coupling parameters will be discussed, as well as the question of the invariance for the coefficients of the $\beta$-functions.

Equivalence under a renormalization group transformation requires (2.1) and (2.2) to satisfy the differential equations

$$
\begin{gathered}
\beta_{1}^{\prime}=\frac{\partial g^{\prime 12}}{\partial g^{2}} \beta_{1}+\frac{\partial g^{\prime 12}}{\partial \lambda} \beta_{2}, \\
\beta_{2}^{\prime}=\frac{\partial \lambda^{\prime}}{\partial \lambda} \beta_{2}+\frac{\partial \lambda^{\prime}}{\partial g^{2}} \beta_{1} .
\end{gathered}
$$

$\beta_{1}^{\prime}, \beta_{2}^{\prime}$ denote the new $\beta$-functions in terms of the new variables,

$$
\begin{gathered}
\beta_{1}^{\prime}=b_{1}^{\prime} g^{14}+\sum_{n=3}^{\infty} \sum_{m=0}^{n-1} b_{n-m, m}^{\prime} g^{\prime 2(n-m)} \lambda^{m}, \\
\beta_{2}^{\prime}=c_{1}^{\prime} \lambda^{\prime 2}+c_{2}^{\prime} \lambda^{\prime} g^{\prime 2}+c_{3}^{\prime} g^{\prime 4}+\sum_{n=3}^{\infty} \sum_{m=0}^{n} c_{n-m, m}^{\prime} \lambda^{\prime n-m} g^{\prime 2 n} .
\end{gathered}
$$

Comparing coefficients in second order $g^{4}, g^{2} \lambda, \lambda^{2}$ yields the invariance of all second order coefficiients

$$
b_{1}^{\prime}=b_{1}, \quad c_{1}^{\prime}=c_{1}, \quad c_{2}^{\prime}=c_{2}, \quad c_{3}^{\prime}=c_{3} .
$$


Comparing third order coefficients of $g^{6}, g^{4} \lambda, g^{2} \lambda^{2}$ in (2.3) we find

$$
\begin{aligned}
& b_{30}^{\prime}=b_{30}+a_{11} c_{3}, \quad b_{21}^{\prime}=b_{21}+a_{11}\left(c_{2}-b_{1}\right), \\
& b_{12}^{\prime}=b_{12}+a_{11} c_{1} .
\end{aligned}
$$

From the third order of (2.4) we get

$$
\begin{aligned}
& c_{30}^{\prime}=c_{30}, \\
& c_{21}^{\prime}=c_{21}+c_{2}\left(d_{20}-a_{11}\right)-c_{3} d_{11}, \\
& c_{12}^{\prime}=c_{12}+2 c_{3}\left(d_{20}-a_{11}\right)+b_{1} d_{11}-a_{20} c_{2}, \\
& c_{03}^{\prime}=c_{03}+c_{3}\left(d_{11}-2 a_{20}\right) .
\end{aligned}
$$

Hence the invariants in third order are

$$
c_{30} \lambda^{3}
$$

and the combination

$$
b_{30}-\frac{c_{3}}{c_{1}} b_{12} .
$$

At this stage we do not dispose of the second order coefficients $a_{20}, a_{11}, d_{20}, d_{11}$ of the transformations (2.1) and (2.2). Instead we try to use them to make all coefficients of $\beta_{1}^{\prime}, \beta_{2}^{\prime}$ in fourth and higher order vanish.

Six more parameters $a_{30}, a_{21}, a_{12}, d_{30}, d_{21}$, and $d_{12}$ enter the fourth order check of the conditions (2.3) and (2.4). Together with the four parameters left over from the third order there are ten parameters available for the purpose of making the fourth order coefficients in $\beta_{1}^{\prime}, \beta_{2}^{\prime}$ vanish. By comparing the coefficients of $g^{8}$, $g^{6} \lambda, g^{4} \lambda^{2}, g^{2} \lambda^{3}$ in (2.3) and of $\lambda^{4}, \lambda^{3} g^{2}, \lambda^{2} g^{4}, \lambda g^{6}, g^{8}$ in (2.4) one obtains nine constraints if all fourth order terms in $\beta_{1}^{\prime}, \beta_{2}^{\prime}$ are set zero. Since there is one variable more than there are constraints it is possible to eliminate the fourth order terms of the $\beta$-functions - apart from exceptional situations.

In fifth order there are eight more parameters in (2.1) and (2.2) together with one parameter left over from fourth order. On the other hand there are eleven constraints if the fifth order coefficients of $\beta_{1}^{\prime}, \beta_{2}^{\prime}$ are required to vanish. Hence in general the available parameters are overdetermined.

In $n^{\text {th }}$ order we get $2(n-1)$ new parameters of the transformations as compared to $2 n+1$ new constraints. Hence it appears impossible to eliminate all fifth and higher order terms in the $\beta$-functions for the general case of two variables.

This does not preclude the possibility of rendering the $\beta$-functions polynomial in special situation. We have therefore checked the supersymmetric case separately in which all powers $g^{2 n}$ in $\beta_{2}$ are absent. This simplification makes it indeed possible to eliminate all fourth and fifth order terms. In sixth order, however, the available parameters are overdetermined. In the $n^{\text {th }}$ order there are $2(n-1)$ new parameters for $2 n$ new constraints.

In conclusion we remark that there are three third order invariants in the supersymmetric case, namely

$$
b_{30} g^{6}, \quad c_{30} \lambda^{3}
$$


and the combination

$$
b_{21}+\frac{b_{1}-c_{2}}{c_{1}} b_{12} \text {. }
$$

\section{Construction of Asymptotic Expansions}

We first study the possibility of power series expansions.

$$
\bar{\lambda}=a_{0}+a_{1} \bar{g}^{2}+a_{2} \bar{g}^{4}+\ldots .
$$

Asymptotic freedom (1.2) requires $a_{0}=0$. Comparing coefficients of $\bar{g}^{4}$ in the differential Eq. (1.11) we find for $a_{1}$ the condition

$$
c_{1} a_{1}^{2}+\left(c_{2}-b_{1}\right) a_{1}+c_{3}=0 .
$$

$a_{1}$ can only be real if $\Delta \geqq 0$ which will be assumed in the work that follows.

We begin with the case $\Delta>0$, or equivalently $\xi \neq 0$. Then there are two distinct solutions for

$$
a_{1}=\varrho_{+} \text {or } \varrho_{-} \text {with } \varrho_{+}>\varrho_{-} .
$$

Comparing the coefficients of $g^{2 n+2}$ in (1.11) with $n=2,3, \ldots$ we find the condition

$$
\left(b, n-2 c_{1} a_{1}-c_{2}\right) a_{n}=E_{n}
$$

for $a_{n}$. $E_{n}$ only depends on lower order coefficients $(m<n)$. If for all $n=2,3, \ldots$ the expression

$$
b_{1} n-2 c_{1} a_{1}-c_{2} \neq 0
$$

does not vanish, all coefficients $a_{n}$ are uniquely determined. A value $n=k$ satisfying

$$
b_{1} k-2 c_{1} a_{1}-c_{2}=0
$$

may directly be related to $\xi$ by

$$
\begin{array}{lll}
\xi=k-1 & \text { if } & a_{1}=\varrho_{-}, \\
\xi=1-k & \text { if } & a_{1}=\varrho_{+},
\end{array}
$$

with $\xi$ defined by (1.15). Hence we arrive at the following statement: If $\xi$ is not a positive or negative integer the differential Eq. (1.11) can be solved by two power series,

$$
\begin{aligned}
& \bar{\lambda}_{+}=\varrho_{+} \bar{g}^{2}+\sum_{n=2}^{\infty} a_{+n} \bar{g}^{2 n}, \\
& \bar{\lambda}_{-}=\varrho_{-} \bar{g}^{2}+\sum_{n=2}^{\infty} a_{-n} \bar{g}^{2 n} .
\end{aligned}
$$

Moreover (3.8) exists for positive integral $\xi,(3.9)$ exists for negative integral $\xi$. If $\xi$ is a positive integer and $E_{\xi+1}=0$ also (3.9) exists. In that case $a_{\xi+1}$ is not restricted by (3.4), hence may be an arbitrary constant. Similarly (3.8) exists with arbitrary $a_{1-\xi}$ if $\xi$ is a negative integer and $E_{1-\xi}=0$. 
In the case $\Delta=0$ (equivalently $\xi=0$ ) the two expansions (3.8) and (3.9) coincide. The construction is unique since (3.6) can only be solved by $k=\xi+1=1$.

This completes the construction of the power series solutions of (1.11). They represent the special solutions which may be constructed. In addition they also provide the general solution if $\xi$ is an integer and $E_{\xi+1}$ or $E_{1-\xi}$ respectively vanishes.

Logarithms occur if $\xi= \pm 1, \pm 2, \ldots$ and $E_{n} \neq 0$ with $n=\xi+1$ or $1-\xi$, respectively. Then (3.6) holds and (3.4) has no solution $a_{n}$. The difficulty can be resolved by adding a logarithmic term

$$
\bar{\lambda}=a_{1} \bar{g}^{2}+\ldots+a_{k} \bar{g}^{2 k}+d_{k} \bar{g}^{2 k} \ln \bar{g}^{2}+\ldots
$$

The inclusion of the logarithmic term will automatically lead to the general solution with an arbitrary parameter. Comparing the coefficients of $\bar{g}^{2 k}$ and $\bar{g}^{2 k} \ln \bar{g}^{2}$ in (1.11), one finds

$$
\begin{aligned}
& \left(b_{1} k-2 c_{1} a_{1}-c_{2}\right) a_{k}=-b_{1} d_{k}+E_{k}, \\
& \left(b_{1} k-2 c_{1} a_{1}-c_{2}\right) d_{k}=0 .
\end{aligned}
$$

Since $b_{1} k-2 c_{1} a_{1}-c_{2}=0$ the two conditions are satisfied by arbitrary $a_{k}$ and $d_{k}=E_{k} / b_{1}$. In higher orders also powers of logarithms occur. Inductively one finds

$$
\begin{gathered}
\bar{\lambda}=\sum_{n=1}^{k-1} a_{n} \bar{g}^{2 n}+a_{k} \bar{g}^{2 k}+d_{k} \bar{g}^{2 k} \ln \bar{g}^{2} \\
+\sum_{\varrho=k+1}^{\infty} \sum_{\sigma} h_{\varrho \sigma} \bar{g}^{2 \varrho} \ln ^{\sigma} \bar{g}^{2}, \\
\left.\left.\begin{array}{l}
k=\xi+1 \\
a_{1}=\varrho_{-}
\end{array}\right\} \text {if } \xi>0, \begin{array}{l}
k=1-\xi \\
a_{1}=\varrho_{+}
\end{array}\right\} \text {if } \quad \xi<0, \quad \xi= \pm 1, \pm 2, \ldots
\end{gathered}
$$

The exponents $\sigma$ of the logarithms are restricted by

$$
1 \leqq \sigma \leqq \frac{\varrho-1}{k-1}
$$

Equation (3.11) represents the general solution in case of integral $\xi \neq 0$.

For $\xi=0$ the solution (1.20) of the approximate systems suggests the ansatz

$$
\bar{\lambda}=\sum_{n=1}^{\infty} a_{n} \bar{g}^{2 n}+\sum_{n=1}^{\infty} \sum_{j=1}^{\infty} d_{n j} \bar{g}^{2 n} \ln ^{-j} \bar{g}^{2}
$$

The logarithmic terms do not affect the recursion formulae of the $a_{n}$. Therefore the first series in (3.12) is the power series expansion of the stable solution. In particular

$$
a_{1}=\varrho_{ \pm}=\frac{b_{1}-c_{2}}{2 c_{1}} .
$$

For the logarithmic terms of order $g^{2}$ one finds

$$
d_{11}=0 \quad \text { or } \quad d_{11}=-\frac{b_{1}}{c_{1}} .
$$


The solution $d_{11}=0$ leads back to the power series expansion since it implies that all $d_{n j}=0$. In the other case $d_{12}$ is arbitrary and all $d_{1 j}(j \geqq 3)$ are unique. The logarithmic terms of order $g^{2}$ can be summed up in closed form by

$$
\sum_{j=1}^{\infty} d_{1 j} \bar{g}^{2} \ln ^{-j} \bar{g}^{2}=-\frac{b_{1}}{c_{1}} \frac{\bar{g}^{2}}{\ln g^{2}+A},
$$

with the integration constant $A$. This follows by making the ansatz

$$
\bar{\lambda}=\varrho\left(\ln \bar{g}^{2}\right) \bar{g}^{2}+o\left(\bar{g}^{4}\right),
$$

which leads to the differential equation

$$
b_{1} \frac{d \varrho}{d \ln \bar{g}^{2}}=c_{1} \varrho^{2}+\left(c_{2}-b_{1}\right) \varrho+c_{3},
$$

with the solutions

$$
\varrho=\frac{b_{1}-c_{2}}{2 c_{1}}-\frac{b_{1}}{c_{1}} \frac{1}{\ln \bar{g}^{2}+A} \quad \text { or } \quad \varrho=\frac{b_{1}-c_{2}}{2 c_{1}} .
$$

The coefficients $d_{n j}(n \geqq 2)$ are uniquely determined by recursion formulae of the form

$$
(n-1) b_{1} d_{n j}=E_{n j},
$$

where $E_{n j}$ is a function of lower order coefficients.

With these results the general solution may be written in the form

$$
\bar{\lambda}=\bar{\lambda}_{ \pm}-\frac{b_{1}}{c_{1}} \frac{\bar{g}^{2}}{\ln \bar{g}^{2}+A}+\sum_{n=2}^{\infty} \sum_{j=1}^{\infty} d_{n j} \bar{g}^{2 n} \ln ^{-j} \bar{g}^{2}, \quad \xi=0,
$$

with the power series (3.8) and (3.9) for $\bar{\lambda}_{ \pm}$.

It remains to construct the general solution for non-integral $\xi$. As suggested by the solution (1.14) of the approximate system we include a term

$$
d_{11} g^{2 k}
$$

where $k=\xi+1$ if $\xi>0, k=1-\xi$ if $\xi<0$. Comparing coefficients in the order $g^{2(k+1)}$ of the differential Eq. (1.11) of $\bar{\lambda}$, one finds

$$
\left(k b_{1}-2 a_{1} c_{1}-c_{2}\right) d_{11}=0 .
$$

We have

$$
k b_{1}-2 a_{1} c_{1}-c_{2}=0 \quad \text { if } \quad \xi>0, a_{1}=\varrho_{-} \quad \text { or } \quad \xi<0, a_{1}=\varrho_{+} .
$$

Hence for $\xi>0$ a term (3.17) with arbitrary coefficient $d_{11}$ may be included in the expansion starting with $\varrho_{-} \bar{g}^{2}$. Similarly for $\xi<0$ when the expansion starting with $\varrho_{+} \bar{g}^{2}$ is used.

When the expansion including the term (3.17) is inserted into the differential Eq. (1.11) terms of the form

$$
d_{m n} \bar{g}^{2(m|\xi|+n)}, \quad m, n=1,2, \ldots
$$


are generated in the expansion of $\bar{\lambda}$. If $\xi$ is rational we write

$$
|\xi|=\frac{p}{q}
$$

as the ratio of relative prime integers $p$ and $q$. The expansion

$$
\bar{\lambda}=\sum_{n=1}^{\infty} a_{n} \bar{g}^{2 n}+\sum_{m=1}^{q-1} \sum_{n=1}^{\infty} d_{m n} \bar{g}^{2(m|\xi|+n)} \quad \text { if } \xi \text { is rational }
$$

or

$$
\bar{\lambda}=\sum_{n=1}^{\infty} a_{n} \bar{g}^{2 n}+\sum_{m=1}^{\infty} \sum_{n=1}^{\infty} d_{m n} \bar{g}^{2(m|\xi|+n)} \quad \text { if } \xi \text { is irrational }
$$

solves the differential equation provided the coefficients satisfy

$$
\begin{gathered}
a_{1} \begin{cases}=\varrho_{-} & \text {if } \quad \xi>0, \\
=\varrho_{+} & \text {if } \quad \xi<0,\end{cases} \\
\left(b_{1}(|\xi|+1)-2 a_{1} c_{1}-c_{2}\right) d_{11}=0,
\end{gathered}
$$

and recursion formulae of the type

$$
\begin{gathered}
\left(b_{1} n-2 a_{1} c_{1}-c_{2}\right) a_{n}=E_{n}, \\
\left(b_{1}(m|\xi|+n)-2 a_{1} c_{1}-c_{2}\right) d_{m n}=E_{m n} .
\end{gathered}
$$

The inhomogeneous terms $E_{n}$ and $E_{m n}$ only depend on lower order coefficients. The coefficients $a_{n}$ are uniquely determined by (3.27). Since $b_{1}(m|\xi|+n)$ $-2 a_{1} c_{1}-c_{2}=0$ only for $m=n=1$, the coefficients $d_{m n}$ are also uniquely determined once the arbitrary value of $d_{11}$ is given. For rational $\xi$ the coefficients $a_{n}$ in general involve $d_{11}$. If $\xi$ is irrational the coefficients $a_{n}$ are not affected by the value of $d_{11}$. Then the first sum in (3.23) represents the power series solution (3.8) or (3.9) respectively.

For the discussion of the leading asymptotic behavior of $\bar{\lambda}$ we assume for simplicity that the roots $\varrho_{ \pm}$are non-vanishing. The supersymmetric case for which $\varrho_{-}=0$ will be treated separately in the following section. By (3.8), (3.9), (3.11), (3.16), and (3.23) the asymptotic behavior of the solutions obtained is

$$
\bar{\lambda}_{+} \simeq \varrho_{+} \bar{g}^{2}, \quad \bar{\lambda}_{-} \simeq \varrho_{-} \bar{g}^{2}, \quad \bar{\lambda} \simeq \varrho_{-} \bar{g}^{2} \text { if } \xi \geqq 0, \quad \bar{\lambda} \simeq \varrho_{+} \tilde{g}^{2} \text { if } \xi \leqq 0,
$$

where $\bar{\lambda}$ denotes the general solution. A particular solution $\bar{\lambda}_{0}$ is called stable if for almost all solutions

$$
\lim _{g \rightarrow 0} \frac{\bar{\lambda}}{\bar{\lambda}_{0}}=1 .
$$

According to (3.24) $\bar{\lambda}_{-}$is stable for $\xi \geqq 0$ and $\bar{\lambda}_{+}$is stable for $\xi \leqq 0 . \bar{\lambda}_{+}$is unstable for $\xi>0$ and $\bar{\lambda}_{-}$unstable for $\xi<0$.

In conclusion we discuss the question of asymptotic freedom. Inserting any of the expansions of $\bar{\lambda}$ back into the differential Eq. (1.3) of $\bar{g}$ with respect to $u$ we obtain

$$
u \frac{\partial \bar{g}^{2}}{\partial u}=\beta_{1}\left(\bar{g}^{2}, \bar{\lambda}\left(\bar{g}^{2}\right)\right)\left\{\begin{array}{lll}
<0 & \text { if } & b_{1}<0 \\
>0 & \text { if } & b_{1}>0
\end{array}\right.
$$


for sufficiently small values of $\bar{g}^{2}$. Hence $\Delta \geqq 0$ and $b_{1}<0$ are necessary conditions for $\lim _{u \rightarrow \infty} \bar{g}=0$ and $\lim _{u \rightarrow \infty} \bar{\lambda}=0$. On the other hand if $b_{1}$ is negative, the solution

$$
u=\exp \int_{g^{2}}^{\bar{g}^{2}} \frac{d x}{\beta_{1}(x, \bar{\lambda}(x))} \quad\left(g^{2}, \bar{g}^{2} \text { sufficiently small }\right)
$$

implies $\bar{g} \rightarrow 0$ for $u \rightarrow \infty$. Hence

With this also

$$
\lim _{u \rightarrow \infty} \bar{g}=0 \quad \text { if } \quad \Delta \geqq 0 \quad \text { and } \quad b_{1}<0 .
$$

$$
\lim _{u \rightarrow \infty} \bar{\lambda}=0
$$

follows for all expansions. This result seems to indicate that the conditions

$$
\Delta \geqq 0, b_{1}<0
$$

are not only necessary but also sufficient for asymptotic freedom. However, for models involving two independent coupling parameters the full range in both variables is not covered by the asymptotic behavior for large Euclidean momenta. For instance, if the roots $\varrho_{ \pm}$do not vanish the ratio $\bar{\lambda} / \bar{g}^{2}$ approaches a nonvanishing value. Hence only for values of $\lambda$ and $g^{2}$ in a sufficient neighborhood of the line $\lambda=\varrho_{ \pm} g^{2}$ asymptotic freedom is guaranted. Though an initial domain of coupling parameters can be enlarged by the equivalence transformations of the renormalization group non-perturbative effects may restrict the ratio $\lambda / g^{2}$ so that asymptotic freedom does not hold. A most obvious restriction of this kind was first found by Browne, O'Raifeartaigh, and Sherry for supersymmetric interactions [6]. We will now give a generalization of this restriction to the class of models considered here. In order to simplify the following discussion we assume $c_{1}>0$ as is usually the case. Then the necessary condition $b_{1}<0$ implies $\xi \geqq 0$, according to the definition (1.15). A model with $\lambda>0$ cannot be asymptotically free if $\varrho_{-} \leqq \varrho_{+}<0$. If $\varrho_{-}<0$, but $\varrho_{+} \geqq 0$, only the unstable mode of the model corresponding to the solution $\bar{\lambda}=\bar{\lambda}_{+}$can be asymptotically free.

\section{Supersymmetric Case ${ }^{2}$}

For supersymmetric interactions $\beta_{2}$ vanishes if $\lambda=0$. Therefore $\bar{\lambda} \equiv 0$ is always a solution of the differential equation (1.11). This trivial solution corresponds to the case $\lambda=0$ of no interaction. For the following discussion we exclude the noninteracting case and require $\lambda=h^{2}>0 . c_{1}>0$ is assumed throughout this section. Since $c_{3}=0$ the roots of (1.16) are

$$
\varrho_{ \pm}=0, \frac{b_{1}-c_{2}}{c_{1}}
$$

and $\Delta \geqq 0$ is always satisfied. If the root $\left(b_{1}-c_{2}\right) / c_{1}$ were negative the general solution (3.11) would become negative for large Euclidean momenta in contradiction to $\lambda>0$. Hence

$$
\begin{gathered}
\frac{b_{1}-c_{2}}{c_{1}} \geqq 0, \quad \varrho_{+}=\frac{b_{1}-c_{2}}{c_{1}}, \quad \varrho_{-}=0, \\
\xi=-\frac{c_{1}}{b_{1}}\left(\varrho_{+}-\varrho_{-}\right)=\frac{c_{2}}{b_{1}}-1 .
\end{gathered}
$$

2 We consider the class of models studied in [6] 
By a similar argument we can exclude $\xi=0$, which is equivalent to $\Delta=0$ or $c_{2}=b_{1}$. Then the leading term of the general solution (3.16) takes the form

$$
\bar{\lambda}=-\frac{b_{1}}{c_{1}} \frac{\bar{g}^{2}}{\ln \bar{g}^{2}+A}+o\left(\bar{g}^{4}\right) .
$$

Since $b_{1}<0$ and $c_{1}>0$, this becomes negative for sufficiently large $\left|k^{2}\right|$ if asymptotic freedom holds with $\bar{g} \rightarrow 0$ for $\left|k^{2}\right| \rightarrow \infty$. The stable solution (3.8) and (3.9) as the only power series expansion possible for $\xi=0$ must reduce to the trivial solution $\bar{\lambda}=0$. Hence for the interacting case $\lambda>0$ a model can only be asymptotically free if

$$
b_{1}<0 \text { and } \xi>0 \text { or equivalently } c_{2}<b_{1}<0 .
$$

We will now simplify the general solutions (3.11), (3.22), and (3.23) for the supersymmetric case. We begin with the case $\xi=1,2, \ldots$ and show that logarithms are absent in (3.11), as well as terms of order less than $g^{2(\xi+1)}$. If $a_{1}=\varrho_{-}=0$. Assuming $a_{1}=\ldots=a_{j-1}=0$, it follows $\left(c_{2}-j b_{1}\right) a_{j}=0$. Hence $a_{j}=0$ if $j \leqq \xi$ and $a_{\xi+1}$ arbitrary. Thus (3.11) reduces to the power series

$$
\bar{\lambda}=\sum_{j=\xi+1}^{\infty} a_{j} g^{2 j}, \quad \xi=1,2, \ldots ; \quad a_{\xi+1} \text { arbitrary } .
$$

If $\xi$ is irrational the expansion (3.23) becomes

$$
\bar{\lambda}=\sum_{m=1}^{\infty} \sum_{n=1}^{\infty} d_{m n} \bar{g}^{2(m \xi+n)}, \quad \xi \text { irrational, } d_{11} \text { arbitrary }
$$

since the coefficients $a_{n}$ are the same as for the solution $\bar{\lambda}_{-}$which vanishes in this case.

$$
\text { For rational } \xi, \quad \xi=\frac{p}{q}>0
$$

with $p$ and $q$ relative prime integers. The first integral power generated in the expansion of $\bar{\lambda}$ is $\bar{g}^{2 p+2}$ so that (3.22) becomes

$$
\bar{\lambda}=\sum_{n=p+1}^{\infty} a_{n} \bar{g}^{2 n}+\sum_{m=1}^{a-1} \sum_{n=1}^{\infty} d_{m n} \bar{g}^{2(m \xi+n)}, \quad d_{11} \text { arbitrary }
$$

In all cases the leading behavior of the general solution is given by $\bar{\lambda} \simeq a \bar{g}^{2(\xi+1)}$ with arbitrary $a$. In contradistinction the unstable solution (3.8) is of order

$$
\bar{\lambda}_{+} \simeq \varrho_{+} \bar{g}^{2}, \quad \varrho_{+}=\frac{b_{1}-c_{2}}{c_{1}}>0 .
$$

\section{References}

1. 't Hooft, G.: Marseille conference on renormalization of Yang-Mills fields and applications to particle physics 1972 (unpublished)

2. Gross, D.J., Wilczek, F.: Ultraviolet behavior of non-abelian gauge theories. Phys. Rev. Lett. 30, 1343-1346 (1973)

3. Politzer, H.D.: Reliable perturbative results for strong interactions? Phys. Rev. Lett. 30, 1346-1349 (1973) 
4. Wilson, K.: Renormalization group and strong interactions. Phys. Rev. D 3, 1818-1846 (1971)

5. Gross, D.J., Wilczek, F.: Asymptotically free gauge theories. I. Phys. Rev. D 8, 3633-3652 (1973)

6. Browne, S., O'Raifeartaigh, L., Sherry, T.: Asymptotic freedom, infrared convergence and supersymmetry. Nucl. Phys. B 99, 150-166 (1975)

7. Kaplunovsky, V.: Absolute asymptotic freedom in SUSY gauge theories. Nucl. Phys. B 211, 297-301 (1983)

8. 't Hooft, G.: Can we make sense of quantum chromodynamics? In: The whys of subnuclear physics (Erice, 1977), pp. 943-950. New York: Plenum Press 1979

Communicated by R. Stora

Received April 13, 1984 